\title{
Information processing in computer-assisted interventions: 5th international conference, 2014
}

\author{
Danail Stoyanov $^{1}$ - D. Louis Collins ${ }^{3}$ - Ichiro Sakuma ${ }^{2}$ - Purang Abolmaesumi ${ }^{5}$. \\ Pierre Jannin ${ }^{4}$
}

Published online: 21 August 2015

(C) CARS 2015

Information processing is an increasingly important tool for surgical interventions. In computer-assisted surgery and interventional radiology, computer systems present valuable information during procedures which is used to help clinical decision-making at the point of treatment. With the paradigm shift towards minimally invasive surgery, where the surgeon or interventionalist has an inherently restricted view of the operating field, this information has a significant influence on how procedures are performed. In fact, surgical instrumentation and technology is increasing in complexity in

$凶$ Danail Stoyanov

danail.stoyanov@ucl.ac.uk

D. Louis Collins

louis.collins@mcgill.ca

Ichiro Sakuma

sakuma@bmpe.t.u-tokyo.ac.jp

Purang Abolmaesumi

purang@ece.ubc.ca

Pierre Jannin

pierre.jannin@univ-rennes1.fr

1 Department of Computer Science, Center for Medical Image Computing, University College London, Front Engineering Building, Gower Street, London WC1E 6BT, UK

2 Medical Device Development and Regulation Research Center, School of Engineering, University of Tokyo, 7-3-1, Hongo, Bunkyo-ku, Tokyo 113-8656, Japan

3 Montreal Neurological Institute, McGill University, 3801 University St., Montreal, QC H4A2B4, Canada

4 MediCIS/LTSI, Inserm/Université de Rennes 1, 2, Avenue du Pr. Léon Bernard, CS 34317, 35043 Rennes, France

5 Department of Electrical and Computer Engineering, University of British Columbia, 2332 Main Mall, Vancouver, BC V6T1Z4, Canada order to reduce access trauma while enhancing surgical precision, and as a result, information processing also has a key role in understanding and optimizing interventional training practices, operating workflow, and ergonomics. Bridging the flow of information and the possibilities for inference between training, diagnosis, intervention, and patient followup, is driven by processing, synthesis, and deduction from the wealth of sensing and imaging technologies that are now driving modern treatment options.

To promote and develop the synergy between the different computer-assisted intervention technologies and capabilities, The 5th International Conference on Information Processing in Computer-Assisted Interventions, IPCAI 2014, was held in Fukuoka, Japan, at the Fukuoka Convention Center on June 28th, 2014. The IPCAI series is a forum for novel technical algorithms and theory, clinical applications, as well as, the hardware and software systems that implement them and their validation. The meeting has a highly cross-disciplinary nature that is critical to developing real solutions to clinically relevant problems, which translate to clinic, improve clinical outcomes, reduce the invasiveness and cost of treatment, and enhance the quality of life postinterventional treatment.

This special issue of IJCARS contains original papers presented at the IPCAI 2014 conference. All presenting authors were invited to submit a revised and extended version of their conference paper, including more methodological detail, further experimental results, and more comprehensive discussion than in the original conference proceedings. All submissions were peer-reviewed by experts form the IPCAI and IJCARS reviewers lists. The accepted papers represent a small but very exciting cross section of the contributions presented at the conference and reflect the breadth of activity currently in the IPCAI and IJCARS community. Given that IPCAI 2014 had only a 50\% accep- 
tance rate and that the IPCAI papers themselves required significant effort and work, the papers accepted and published in this issue really have great content and potential impact.
Acknowledgments The special issue editors would like to thank both the IPCAI and IJCARS reviewers of these papers and IJCARS editorial staff for their efforts in preparing the presentation of these works to the community. 